\title{
A SCHUR MULTIPLIER CHARACTERIZATION OF COARSE EMBEDDABILITY
}

\author{
SØREN KNUDBY AND KANG LI
}

\begin{abstract}
We give a contractive Schur multiplier characterization of locally compact groups coarsely embeddable into Hilbert spaces. Consequently, all locally compact groups whose weak Haagerup constant is 1 embed coarsely into Hilbert spaces, and hence the Baum-Connes assembly map with coefficients is split-injective for such groups.
\end{abstract}

In this note we study coarse embeddability of locally compact groups into Hilbert spaces. An important application of this concept in [16], [13] and [5] is that the Baum-Connes assembly map with coefficients is split-injective for all locally compact groups that embed coarsely into a Hilbert space (see [2] and [15] for more information about the Baum-Connes assembly map). Here, we give a contractive Schur multiplier characterization of locally compact groups coarsely embeddable into Hilbert spaces (see also [6, Theorem 5.3] for the discrete case), and this characterization can be regarded as an answer to the non-equivariant version of $[12$, Question 1.5]. As a result, any locally compact group with weak Haagerup constant 1 embeds coarsely into a Hilbert space and hence the Baum-Connes assembly map with coefficients is split-injective for all these groups.

Let $G$ be a $\sigma$-compact, locally compact group. A (left) tube in $G \times G$ is a subset of $G \times G$ contained in a set of the form

$$
\operatorname{Tube}(K)=\left\{(x, y) \in G \times G \mid x^{-1} y \in K\right\}
$$

where $K$ is any compact subset of $G$. Following [1, Definition 3.6], we say that a map $u$ from $G$ into a Hilbert space $H$ is a coarse embedding if $u$ satisfies the following two conditions:

- for every compact subset $K$ of $G$ there exists $R>0$ such that

$$
(s, t) \in \operatorname{Tube}(K) \Longrightarrow\|u(s)-u(t)\| \leq R ;
$$

- for every $R>0$ there exists a compact subset $K$ of $G$ such that

$$
\|u(s)-u(t)\| \leq R \Longrightarrow(s, t) \in \operatorname{Tube}(K) .
$$

We say that a group $G$ embeds coarsely into a Hilbert space or admits a coarse embedding into a Hilbert space if there exist a Hilbert space $H$ and a coarse embedding $u: G \rightarrow H$. Note that a coarse embedding need not be injective, and we also do not require it to be continuous.

Date: November 7, 2018.

Both authors are supported by ERC Advanced Grant no. OAFPG 247321 and the Danish National Research Foundation through the Centre for Symmetry and Deformation (DNRF92). Mathematics Subject Classification (2010): 43A22, 43A35, 46L80.

Keywords: Coarse embedding, Schur multipliers, Baum-Connes conjecture. 
Every second countable, locally compact group $G$ admits a proper left-invariant metric $d$, which is unique up to coarse equivalence (see [14] and [9]). So the preceding definition is equivalent to Gromov's notion of coarse embeddability of the metric space $(G, d)$ into Hilbert spaces. We refer to [5, Section 3] for more on coarse embeddability into Hilbert spaces for locally compact groups.

A kernel $\varphi: G \times G \rightarrow \mathbb{C}$ is a Schur multiplier if for every bounded operator $A=$ $\left(a_{x, y}\right)_{x, y \in G} \in B\left(\ell^{2}(G)\right)$, the matrix $\left(\varphi(x, y) a_{x, y}\right)_{x, y \in G}$ again defines a bounded operator, denoted $M_{\varphi} A$, on $\ell^{2}(G)$. In this case, it follows from the closed graph theorem that $M_{\varphi}$ in fact defines a bounded operator $B\left(\ell^{2}(G)\right) \rightarrow B\left(\ell^{2}(G)\right)$, and the Schur norm $\|\varphi\|_{S}$ of $\varphi$ is defined to be the operator norm of $M_{\varphi}$.

A kernel $\varphi: G \times G \rightarrow \mathbb{C}$ tends to zero off tubes, if for any $\varepsilon>0$ there is a tube $T \subseteq G \times G$ such that $|\varphi(x, y)|<\varepsilon$ whenever $(x, y) \notin T$. Note that if $\varphi: G \rightarrow \mathbb{C}$ is a function, then $\varphi$ vanishes at infinity (written $\varphi \in C_{0}(G)$ ), if and only if the associated kernel $\widehat{\varphi}: G \times G \rightarrow \mathbb{C}$ defined by $\widehat{\varphi}(x, y)=\varphi\left(x^{-1} y\right)$ tends to zero off tubes.

Theorem 1. Let $G$ be a $\sigma$-compact, locally compact group. The following are equivalent.

(1) $G$ embeds coarsely into a Hilbert space.

(2) There exists a sequence of Schur multipliers $\varphi_{n}: G \times G \rightarrow \mathbb{C}$ such that

- $\left\|\varphi_{n}\right\|_{S} \leq 1$ for every natural number $n$;

- each $\varphi_{n}$ tends to zero off tubes;

- $\varphi_{n} \rightarrow 1$ uniformly on tubes.

If any of these conditions holds, one can moreover arrange that the coarse embedding is continuous and that each $\varphi_{n}$ is continuous.

It is well-known that the notion of coarse embeddability into Hilbert spaces can be characterized by positive definite kernels (see [8, Theorem 2.3] for the discrete case and $[4$, Theorem 1.5] for the locally compact case).

Following [11], $G$ has the weak Haagerup property with constant 1, if there is a sequence of continuous functions $\varphi_{n} \in C_{0}(G)$ convering uniformly to 1 on compact subsets of $G$ and such that the associated kernels $\widehat{\varphi}_{n}: G \times G \rightarrow \mathbb{C}$ are Schur multipliers with $\left\|\widehat{\varphi}_{n}\right\|_{S} \leq 1$.

From Theorem 1 together with [5, Theorem 3.5] we immediately obtain the following.

Corollary 2. If $G$ is a $\sigma$-compact, locally compact group with the weak Haagerup property with constant 1 , then $G$ embeds coarsely into a Hilbert space. If $G$ is moreover second countable, then in particular the Baum-Connes assembly map with coefficients is split-injective.

We now turn to the proof of Theorem 1. It is not hard to see that the countability assumption in [10, Proposition 4.3] is superfluous. We thus record the following (slightly more general) version of [10, Proposition 4.3].

Lemma 3. Let $G$ be a group with a symmetric kernel $k: G \times G \rightarrow[0, \infty)$. The following are equivalent.

(1) For every $t>0$ one has $\left\|e^{-t k}\right\|_{S} \leq 1$. 
(2) There exist a real Hilbert space $\mathcal{H}$ and maps $R, S: G \rightarrow \mathcal{H}$ such that

$$
k(x, y)=\|R(x)-R(y)\|^{2}+\|S(x)+S(y)\|^{2} \quad \text { for every } x, y \in G .
$$

Recall that a kernel $k: G \times G \rightarrow \mathbb{R}$ is conditionally negative definite if $k$ is symmetric $(k(x, y)=k(y, x))$, vanishes on the diagonal $(k(x, x)=0)$ and

$$
\sum_{i, j=1}^{n} c_{i} c_{j} k\left(x_{i}, x_{j}\right) \leq 0
$$

for any finite sequences $x_{1}, \ldots, x_{n} \in G$ and $c_{1}, \ldots, c_{n} \in \mathbb{R}$ such that $\sum_{i=1}^{n} c_{i}=0$. It is well-known that $k$ is conditionally negative definite if and only if there is a function $u$ from $G$ to a real Hilbert space such that $k(x, y)=\|u(x)-u(y)\|^{2}$.

A kernel $k: G \times G \rightarrow \mathbb{C}$ is called proper, if the set $\{(x, y) \in G \times G|| k(x, y) \mid \leq R\}$ is a tube for every $R>0$.

Theorem 1 is contained in Theorem 4 below, which extends both [6, Theorem 5.3] and [4, Theorem 1.5] in different directions. An important ingredient in the proof of Theorem 4 is the following result (which generalizes without change from the second countable case to the $\sigma$-compact case).

Theorem ([5, Theorem 3.4]). Let $G$ be a $\sigma$-compact, locally compact group. The following are equivalent.

(1) The group $G$ embeds coarsely into a Hilbert space.

(2) There is a continuous conditionally negative definite kernel $h: G \times G \rightarrow \mathbb{R}$ which is proper and bounded on tubes.

Theorem 4. Let $G$ be a $\sigma$-compact, locally compact group. The following are equivalent.

(1) The group $G$ embeds coarsely into a Hilbert space.

(2) There exists a sequence of (not necessarily continuous) Schur multipliers $\varphi_{n}: G \times G \rightarrow \mathbb{C}$ such that

- $\left\|\varphi_{n}\right\|_{S} \leq 1$ for every natural number $n$;

- each $\varphi_{n}$ tends to zero off tubes;

- $\varphi_{n} \rightarrow 1$ uniformly on tubes.

(3) There exists a (not necessarily continuous) symmetric kernel $k: G \times G \rightarrow$ $[0, \infty)$ which is proper, bounded on tubes and satisfies $\left\|e^{-t k}\right\|_{S} \leq 1$ for all $t>0$.

(4) There exists a (not necessarily continuous) conditionally negative definite kernel $h: G \times G \rightarrow \mathbb{R}$ which is proper and bounded on tubes.

Moreover, if any of these conditions holds, one can arrange that the coarse embedding in (1), each Schur multiplier $\varphi_{n}$ in (2), the symmetric kernel $k$ in (3) and the conditionally negative definite kernel $h$ in (4) are continuous.

Proof. We show $(1) \Longleftrightarrow(4) \Longleftrightarrow(3) \Longleftrightarrow(2)$.

That (1) implies (4) with $h$ continuous follows directly from [5, Theorem 3.4].

Suppose (4) holds. By the GNS construction there are a real Hilbert space $\mathcal{H}$ and a map $u: G \rightarrow \mathcal{H}$ such that

$$
h(x, y)=\|u(x)-u(y)\|^{2} .
$$


It is easy to check that the assumptions on $h$ imply that $u$ is a coarse embedding. Thus (1) holds.

That (4) implies (3) follows with $k=h$ using Schoenberg's Theorem and the fact that normalized positive definite kernels are Schur multipliers of norm 1. Note also that conditionally negative definite kernels are symmetric and take only nonnegative values.

Suppose (3) holds. We show that (4) holds. From Lemma 3 we see that there are a real Hilbert space $\mathcal{H}$ and maps $R, S: G \rightarrow \mathcal{H}$ such that

$$
k(x, y)=\|R(x)-R(y)\|^{2}+\|S(x)+S(y)\|^{2} \quad \text { for every } x, y \in G .
$$

As $k$ is bounded on tubes, the map $S$ is bounded. If we let

$$
h(x, y)=\|R(x)-R(y)\|^{2},
$$

then it is easily checked that $h$ is proper and bounded on tubes, since $k$ has these properties and $S$ is bounded. It is also clear that $h$ is conditionally negative definite. Thus (4) holds.

If (3) holds, we set $\varphi_{n}=e^{-k / n}$ when $n \in \mathbb{N}$. It is easy to check that the sequence $\varphi_{n}$ has the desired properties so that (2) holds.

Finally, suppose (2) holds. We verify (3). Essentially, we use the same standard argument as in the proof of [11, Proposition 4.4] and [3, Theorem 2.1.1].

Since $G$ is locally compact and $\sigma$-compact, it is the union of an increasing sequence $\left(U_{n}\right)_{n=1}^{\infty}$ of open sets such that the closure $K_{n}$ of $U_{n}$ is compact and contained in $U_{n+1}$ (see [7, Proposition 4.39]). Fix an increasing, unbounded sequence $\left(\alpha_{n}\right)$ of positive real numbers and a decreasing sequence $\left(\varepsilon_{n}\right)$ tending to zero such that $\sum_{n} \alpha_{n} \varepsilon_{n}$ converges. By assumption, for every $n$ we can find a Schur multiplier $\varphi_{n}$ tending to zero off tubes and such that $\left\|\varphi_{n}\right\|_{S} \leq 1$ and

$$
\sup _{(x, y) \in \operatorname{Tube}\left(K_{n}\right)}\left|\varphi_{n}(x, y)-1\right| \leq \varepsilon_{n} / 2 .
$$

Upon replacing $\varphi_{n}$ by $\left|\varphi_{n}\right|^{2}$ one can arrange that $0 \leq \varphi_{n} \leq 1$ and

$$
\sup _{(x, y) \in \operatorname{Tube}\left(K_{n}\right)}\left|\varphi_{n}(x, y)-1\right| \leq \varepsilon_{n} .
$$

Define kernels $\psi_{i}: G \times G \rightarrow[0, \infty[$ and $\psi: G \times G \rightarrow[0, \infty[$ by

$$
\psi_{i}(x, y)=\sum_{n=1}^{i} \alpha_{n}\left(1-\varphi_{n}(x, y)\right), \quad \psi(x, y)=\sum_{n=1}^{\infty} \alpha_{n}\left(1-\varphi_{n}(x, y)\right) .
$$

It is easy to see that $\psi$ is well-defined, bounded on tubes and $\psi_{i} \rightarrow \psi$ pointwise (even uniformly on tubes, but we do not need that).

To see that $\psi$ is proper, let $R>0$ be given. Choose $n$ large enough such that $\alpha_{n} \geq 2 R$. As $\varphi_{n}$ tends to zero off tubes, there is a compact set $K \subseteq G$ such that $\left|\varphi_{n}(x, y)\right|<1 / 2$ whenever $(x, y) \notin \operatorname{Tube}(K)$. Now if $\psi(x, y) \leq R$, then $\psi(x, y) \leq \alpha_{n} / 2$, and in particular $\alpha_{n}\left(1-\varphi_{n}(x, y)\right) \leq \alpha_{n} / 2$, which implies that $1-\varphi_{n}(x, y) \leq 1 / 2$. We have thus shown that

$\{(x, y) \in G \times G \mid \psi(x, y) \leq R\} \subseteq\left\{(x, y) \in G \times G \mid 1-\varphi_{n}(x, y) \leq 1 / 2\right\} \subseteq \operatorname{Tube}(K)$, and $\psi$ is proper. 
We now show that $\left\|e^{-t \psi}\right\|_{S} \leq 1$ for every $t>0$. Since $\psi_{i}$ converges pointwise to $\psi$, it will suffice to prove that $\left\|e^{-t \psi_{i}}\right\|_{S} \leq 1$, because the set of Schur multipliers of norm at most 1 is closed under pointwise limits. Since

$$
e^{-t \psi_{i}}=\prod_{n=1}^{i} e^{-t \alpha_{n}\left(1-\varphi_{n}\right)}
$$

it is enough to show that $e^{-t \alpha_{n}\left(1-\varphi_{n}\right)}$ has Schur norm at most 1 for each $n$. And this is clear:

$$
\left\|e^{-t \alpha_{n}\left(1-\varphi_{n}\right)}\right\|_{S}=e^{-t \alpha_{n}}\left\|e^{t \alpha_{n} \varphi_{n}}\right\|_{S} \leq e^{-t \alpha_{n}} e^{t \alpha_{n}\left\|\varphi_{n}\right\|_{S}} \leq 1 .
$$

The only thing missing is that $\psi$ need not be symmetric. Put $k=\psi+\breve{\psi}$ where $\breve{\psi}(x, y)=\psi(y, x)$. Clearly, $k$ is symmetric, bounded on tubes and proper. Finally, for every $t>0$

$$
\left\|e^{-t k}\right\|_{S} \leq\left\|e^{-t \psi}\right\|_{S}\left\|e^{-t \check{\psi}}\right\|_{S} \leq 1
$$

since $\|\check{\varphi}\|_{S}=\|\varphi\|_{S}$ for every Schur multiplier $\varphi$.

Finally, the statements about continuity follow from [5, Theorem 3.4] and the explicit constructions used in our proof of $(1) \Longrightarrow(4) \Longrightarrow(3) \Longrightarrow(2)$.

\section{REFERENCES}

[1] Claire Anantharaman-Delaroche. Amenability and exactness for dynamical systems and their $C^{*}$-algebras. Trans. Amer. Math. Soc., 354(10):4153-4178 (electronic), 2002.

[2] Paul Baum, Alain Connes, and Nigel Higson. Classifying space for proper actions and $K$ theory of group $C^{*}$-algebras. In $C^{*}$-algebras: 1943-1993 (San Antonio, TX, 1993), volume 167 of Contemp. Math., pages 240-291. Amer. Math. Soc., Providence, RI, 1994.

[3] Pierre-Alain Cherix, Michael Cowling, Paul Jolissaint, Pierre Julg, and Alain Valette. Groups with the Haagerup property, volume 197 of Progress in Mathematics. Birkhäuser Verlag, Basel, 2001. Gromov's a-T-menability.

[4] Steven Deprez and Kang Li. Permanence properties of property A and coarse embeddability for locally compact groups. Preprint, arXiv:1403.7111, 2014.

[5] Steven Deprez and Kang Li. Property A and uniform embedding for locally compact groups. Preprint, to appear in J. Noncommut. Geom., 2015.

[6] Zhe Dong and Zhong-Jin Ruan. A Hilbert module approach to the Haagerup property. Integral Equations Operator Theory, 73(3):431-454, 2012.

[7] Gerald B. Folland. Real analysis. Pure and Applied Mathematics (New York). John Wiley \& Sons Inc., New York, second edition, 1999. Modern techniques and their applications, A Wiley-Interscience Publication.

[8] Erik Guentner and Jerome Kaminker. Exactness and the Novikov conjecture. Topology, 41(2):411-418, 2002.

[9] Uffe Haagerup and Agata Przybyszewska. Proper metrics on locally compact groups, and proper affine isometric actions on Banach spaces. Preprint, arXiv:math/0606794, 2006.

[10] Søren Knudby. Semigroups of Herz-Schur multipliers. J. Funct. Anal., 266(3):1565-1610, 2014.

[11] Søren Knudby. The weak Haagerup property. Preprint, to appear in Trans. Amer. Math. Soc., 2015.

[12] Søren Knudby and Kang Li. Approximation properties of simple Lie groups made discrete. Preprint, to appear in J. Lie Theory, 2015.

[13] G. Skandalis, J. L. Tu, and G. Yu. The coarse Baum-Connes conjecture and groupoids. Topology, 41(4):807-834, 2002.

[14] Raimond A. Struble. Metrics in locally compact groups. Compositio Math., 28:217-222, 1974.

[15] Alain Valette. Introduction to the Baum-Connes conjecture. Lectures in Mathematics ETH Zürich. Birkhäuser Verlag, Basel, 2002. From notes taken by Indira Chatterji, With an appendix by Guido Mislin. 
[16] Guoliang Yu. The coarse Baum-Connes conjecture for spaces which admit a uniform embedding into Hilbert space. Invent. Math., 139(1):201-240, 2000.

Department of Mathematical Sciences, University of Copenhagen,

Universitetsparken 5, DK-2100 Copenhagen $\varnothing$, Denmark

E-mail address: knudby@math.ku.dk

Department of Mathematical Sciences, University of Copenhagen,

Universitetsparken 5, DK-2100 Copenhagen $\varnothing$, Denmark

E-mail address: kang.li@math.ku.dk 